\title{
S64-02
}

\section{ADDICTION SERVICES IN CYPRUS: RESULTS OF THE EU TWINNING PROJECT EVALUATION}

A. Agorastos ${ }^{1}$, H. Zurhold ${ }^{2}$, A. Petridou ${ }^{3}$, C. Haasen ${ }^{4}$

${ }^{1}$ Clinic for Psychiatry and Psychotherapy, Centre of Psychosocial Medicine, ${ }^{2}$ Centre for Interdiscilinary Addiction Research (ZIS), UKE, University Medical Centre Hamburg-Eppendorf, Hamburg, Germany, ${ }^{3}$ PERSEAS Counselling Centre for Adolescents and Family, Cyprus Mental Health Services, Nicosia, Cyprus, ${ }^{4}$ Clinic for Psychiatry and Psychotherapy, Centre of Psychosocial Medicine, UKE, University Medical Centre Hamburg-Eppendorf, Centre for Interdisciplinary Addictio Research (ZIS), Hamburg, Germany

In April 2007 a European twinning project started between Cyprus and Germany, in order to assist Cyprus as a new Candidate Country in the implementation and harmonisation with the European Community's legislation, by evaluating the governmental drug services in Cyprus and their coordination and promoting the improvement and introduction of new drug treatment services. A field investigation studied parameters as population in need, treatment demand and coverage, as well as high risk patterns and their trend over the last years, showing an individual place of Cyprus in the international drug ranking, with the highest prevalence of cannabis and second highest on ecstasy abuse and one of the smallest in opiates and amphetamine consumption. Nevertheless heroin remains the primary drug of abuse in persons seeking treatment, although Cyprus was until 2008 the only country of the European Union without a substitution treatment. Furthermore this project studies in detail the current availability of the governmental drug facilities, their structure and coordination as well as the legal framework in which they operate, showing a still adapting drug care system, trying to keep up with the increasing special needs of the Cypriot population, but offering the presuppositions for an efficient and good functioning drug care system in the future. 